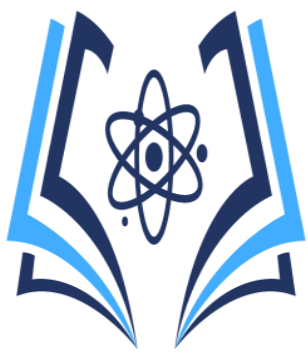

\title{
Abordaje Integral para la intervención de las conductas adictivas en Adolescentes del cantón Jipijapa
}

\section{Prevention and care of addictive behaviors in Adolescents of the Jipijapa canton}

\section{Conductas adictivas en Adolescentes}

\author{
Mg Delia Georgina Bravo Bonoso ${ }^{(1)}$ \\ Mg. Doris Susana Delgado Bernal ${ }^{(2)}$ \\ Mg. Bárbara Miladys Placencia López ${ }^{(3)}$ \\ Mg. Sidar Edgardo Solórzano Solórzano ${ }^{(4)}$ \\ ${ }^{1}$ Universidad Estatal del Sur de Manabí, delia.bravo@unesum.edu.ec, Orcid: https://orcid.org/0000-0003- \\ 4787-8403
}

${ }^{2}$ Universidad Estatal del Sur de Manabí, doris.delgado@unesum.edu.ec, Orcid: https://orcid.org/00000001-5614-2567

${ }^{3}$ Universidad Estatal del Sur de Manabí, miladys.placencia@ unesum.edu.ec, Orcid: https://orcid.org/00000003-4970-2305

${ }^{4}$ Universidad Estatal del Sur de Manabí, sidar.solorzano@unesum.edu.ec, Orcid: https://orcid.org/00000002-6990-8397

Contacto: delia.bravo@unesum.edu.ec

\section{Resumen}

Las drogas son un problema a nivel mundial, muchos adolescentes comienzan a consumirla por curiosidad, influencia del grupo de amigos, depresión $\mathrm{u}$ otros problemas dentro de la sociedad, los riesgos que puede ocasionar el consumo de estas sustancias se transforman en problemas familiares, económicos, muchos de los consumidores llegan a cometer actos ilícitos, dentro de la sociedad para poder adquirir estas sustancias En el presente trabajo de investigación, se analizan los diferentes modelos interpretativos que pretenden comprender y dar explicación a la problemática del consumo de drogas. Para ello, se estudian varias interpretaciones teóricas como: modelo jurídico, modelo de la distribución del consumo, modelo médico tradicional, modelo de reducción del daño, modelo de la privación social, modelo de los factores socio estructurales, modelo de educación para la salud, modelo psicológico individualista y modelo socio ecológico. En cada caso se expone su particular perspectiva interpretativa, sus aportaciones más relevantes y una discusión sobre sus limitaciones. El modelo de abordaje integral para la intervención de conductas adictivas, se presenta como una alternativa de abordaje ante la problemática del 
consumo de drogas y para intervenir eficazmente sobre ella, dada su vocación integradora.

Palabras clave: drogadicción, intervención, familia, dependencia. incidencia, autocontrol.

\section{Sumary}

Drugs are a worldwide problem, many adolescents begin to consume it out of curiosity, influence of the group of friends, depression or other problems within society, the risks that the consumption of these substances can cause are transformed into family and economic problems, many of the consumers come to commit illicit acts within society in order to acquire these substances. In this research work, the different interpretive models that seek to understand and explain the problem of drug use are analyzed. For this, several theoretical interpretations are studied such as: legal model, consumption distribution model, traditional medical model, harm reduction model, social deprivation model, socio-structural factors model, health education model, model individualistic psychological and socio-ecological model. In each case, its particular interpretative perspective, its most relevant contributions and a discussion about its limitations are exposed. The comprehensive approach model for the intervention of addictive behaviors, is presented as an alternative approach to the problem of drug use and to intervene effectively on it, given its integrating vocation.

Key words: drug addiction, intervention, family, dependence. incidence, self-control.

\section{Introducción}

El consumo de drogas es tan antiguo como la humanidad. Se encuentra en la literatura de Europa, África, Asía reseñas del uso como parte de manifestaciones culturales, además se vislumbra lo que ahora se define como abuso, en esas épocas no era reconocido de forma directa como un asunto a ser analizado e intervenido
ISSN 2773-7705

Periodo. Julio - Diciembre 2021

Vol. 5, Nro. 2, Publicado: 2021-12-31 (Municipio de Medellin- Ministerio de Salud, 2014) En el área de la salud, hace ya muchos años, se reconocen los trastornos adictivos como enfermedades y, en años más recientes, como problemas de salud pública; esto ha permitido que haya un interés mundial creciente en el tema que ha permitido un campo de investigación amplio y ha motivado a los profesionales y a los políticos a dar un trato social más comprensivo para estas problemáticas.

El fenómeno de las drogas a nivel mundial representa una problemática cada vez más compleja, dinámica y multicausal, que genera daños en la salud, en la convivencia social y seguridad ciudadana, pues desintegra a la sociedad y, sobre todo, su núcleo central que es la familia. Esta situación problemática demanda un abordaje integral, multidisciplinario, inmediato y basado en evidencia científica, con el involucramiento activo de la sociedad a través de sus distintos actores. La información de la problemática en nuestra realidad nacional, alerta acerca de aspectos como el consumo de drogas en edades tempranas, el relativo fácil acceso a las mismas y la determinación de las zonas con mayor incidencia del fenómeno socio económico de las drogas.

Las conductas adictivas tienen origen en espacios de interacción donde se propicia el consumo de diferentes sustancias toxicas o el exceso de juegos adictivos, que generan interferencias en los contextos donde se desenvuelven los seres humanos afectando su área familiar, laboral, escolar y social. Para disminuir este fenómeno que se presenta continuamente en la sociedad es importante poder contar con alternativas de prevención de consumo o en los casos específicos de drogodependencia contar con rehabilitación de 
las conductas adictivas (Instituto Provincial de Bienestar Social de Cordoba, 2018).

Las sustancias aditivas son un serio problema de salud pública en el mundo y los países en desarrollo cada vez va en aumento afectando a todas las familias. A partir de los años sesenta, el consumo se transformó en una preocupación mundial, particularmente en los países industrializados sobre todo por la alta cantidad de riesgos que pueden ocasionar a la salud, la economía, a los problemas jurídicos, familiares, teniendo serias implicaciones en la violencia, delincuencia y el terrorismo internacional ( Guaranda Moreira , 2018) este consumo excesivo causa diversos tipos de problemas, dentro de las familias y en la sociedad, pues las personas que la consumen pueden llegar a ser violentas o hacer cosas ilícitas por conseguir estas sustancias.

La prevención de las adicciones y la promoción de la salud de personas consumidoras de sustancias o con conductas de riesgo, aunque son dos hechos distintos entre sí, sus objetivos se superponen. El primero está relacionado con la interacción de las sustancias con el sujeto en un contexto determinado de relación, sobre los que sería necesario intervenir si queremos conseguir una mejora de la vida de las personas (modelo ecológico) y que abarcaría toda acción tendente a evitar o disminuir el consumo de drogas o a paliar los efectos que el consumo puede ocasionar en los consumidores. El segundo se sitúa dentro de un concepto más amplio de promoción de la salud lo que conlleva poner en marcha acciones que apoyen a las personas a adoptar y mantener estilos de vida saludables y a que desarrollen condiciones de vida y ambientes que promocionen la salud, y no se puede entender si no es con la participación de la comunidad. (Tumbaco Quimis, 2018).
ISSN 2773-7705

Periodo. Julio - Diciembre 2021

Vol. 5, Nro. 2, Publicado: 2021-12-31

Más allá de estas consideraciones, la prudencia nos aconseja que debemos realizar siempre una evaluación de las actuaciones que llevemos a cabo y de los resultados obtenidos; así como de monitorizar los cambios experimentados tanto a nivel social como medioambiental para ampliar la evidencia y alentar a mejorar las actuaciones llevadas a cabo en prevención, adaptándolas a cada momento, lugar y población al que van dirigidas.

Desafortunadamente, nos encontramos en muchas ocasiones con profesionales con un alto nivel de capacitación que, inmersos en el quehacer diario, están con frecuencia tan preocupados con los problemas inmediatos, de las personas que tienen un problema adictivo, que se olvidan de poder prestar atención a aquellos que están "bien” (Tena Suck, Castro Martínez, Marín Navarrete, Gómez Romero, \& De la Fuente-Martín, 2018)

La adolescencia es una de las fases de la vida más fascinantes y quizás más complejas, una época en que la gente joven asume nuevas responsabilidades y experimenta una nueva sensación de independencia. Los jóvenes buscan su identidad, aprenden a poner en práctica valores aprendidos en su primera infancia y a desarrollar habilidades que les permitirán convertirse en adultos atentos y responsables. (6) Cuando los adolescentes reciben el apoyo y el aliento de los adultos, se desarrollan de formas inimaginables, convirtiéndose en miembros plenos de sus familias y comunidades $y$ dispuestos a contribuir. Llenos de energía, curiosidad y de un espíritu que no se extingue fácilmente, los jóvenes tienen en sus manos la capacidad de cambiar los modelos de conducta sociales negativos y romper con el ciclo de la violencia y la discriminación que se transmite de generación en generación. 
Con su creatividad, energía y entusiasmo, los jóvenes pueden cambiar el mundo de forma impresionante, logrando que sea un lugar mejor, no sólo para ellos mismos sino también para todos. Por otra parte, en el ámbito familiar se nombran algunos factores como: el modo de enfrentar los problemas a nivel familiar, y dentro de estos, la falta de apoyo religioso y de la búsqueda de apoyo profesional, conjuntamente con las dificultades de comunicación intrafamiliar (Cid Monckton \& Pedrão , 2011); las pautas de interacción familiar, como la comunicación deficiente, los frecuentes conflictos, la baja cohesión, el excesivo castigo o el consumo de los propios padres (Jimenez, Murgui, Estevez, \& Musitu, 2007).

La familia emerge como núcleo desencadenante de conductas de riesgo, si el adolescente la percibe como un ambiente hostil busca un grupo que le brinde seguridad, pero al carecer de una referencia fiable de sus propios atributos, presenta un proceso de desadaptación provocándole el rechazo de otros. Esto deriva en la exposición a factores de riesgo como el consumo de drogas, la promiscuidad y conductas violentas ( Gómez Cobos, 2018).

La universidad en conjunto con el Ministerio de Salud Pública, tienen la obligación ética ineludible de desarrollar los mecanismos que le permitan suministrar a la población afectada por el consumo de drogas, la posibilidad de hallar tratamiento y recuperarse, para reincorporándose de manera adecuada a su entorno familiar, comunitario y social. Por tal razón se está identificando a las personas con consumo problemático, para un abordaje integral que nos permita hallar elementos de carácter científico que propendan por una intervención adecuada, apuntando hacia la mejor calidad de atención
ISSN 2773-7705

Periodo. Julio - Diciembre 2021

Vol. 5, Nro. 2, Publicado: 2021-12-31 posible, accesible a toda la población que así lo requiera.

\section{Material y métodos}

\section{Diseño}

Es un estudio descriptivo, analítico no experimental, desde un enfoque empírico, basándonos en la revisión de la literatura específicamente en él, modelo jurídico, modelo de la distribución del consumo, modelo médico tradicional, modelo de reducción del daño, modelo de la privación social, modelo de los factores socio estructurales, modelo de educación para la salud, modelo psicológico individualista y modelo socio ecológico, mismos que permitirán a su vez un abordaje integral de las conductas adictivas. Este modelo podrá emplearse en las 4 ciudadelas de la periferia urbana del cantón, donde se realizó una barrido casa a casa, identificando posibles adolescentes con consumo problemático.

\section{Resultados}

Según la revisión bibliográfica hemos resumido y adaptado para un abordaje integral a los adolescentes con consumo problemático, se recomienda que se incluyan todos los siguientes puntos dentro de la evaluación clínica.

1. Identificación: datos de filiación (edad, sexo, estado civil, convivencia, nivel académico, residencia, profesión, ocupación, situación laboral, nivel económico, etc.)

2. Situación actual por la que el paciente demanda el tratamiento, incluyendo:

a) Motivo de consulta/demanda de tratamiento: profundizar la razón real que lleva al paciente a buscar ayuda, en donde incluya; problemas económicos judiciales, familiares, laborales, o por voluntad propia y deseo de superar la adicción. 
b) Estado de intoxicación/abstinencia: de la gravedad e intensidad dependerá la facilidad o dificultad de obtener la información.

c) Disposición actual para el cambio: revela la aceptación del paciente con relación a su
Vol. 5, Nro. 2, Publicado: 2021-12-31 enfermedad adictiva, permitiendo orientar los objetivos terapéuticos a futuro. En este acápite se podrá establecer el estadio en el que se encuentra el paciente:

Tabla 1: Estadios en el proceso del cambio y objetivos terapéuticos.

\begin{tabular}{|l|l|l|}
\hline Estadio & Definición & Objetivo terapéutico \\
\hline Pre & $\begin{array}{l}\text { El paciente no es consciente de que su } \\
\text { conducta es problemática y no se plantea } \\
\text { modificarla- }\end{array}$ & $\begin{array}{l}\text { Generar dudas, haciendo ver la } \\
\text { posible relación existente entre su } \\
\text { conducta y las consecuencias } \\
\text { detectada. }\end{array}$ \\
\hline Contemplación & $\begin{array}{l}\text { Paciente consciente de la existencia de un } \\
\text { problema. Comienza a pensar en la } \\
\text { posibilidad de realizar un cambio (aún } \\
\text { existe ambivalencia) }\end{array}$ & $\begin{array}{l}\text { Facilitar el análisis de los pros y los } \\
\text { contras de su conducta }\end{array}$ \\
\hline Preparación & $\begin{array}{l}\text { El paciente toma la decisión de cambiar } \\
\text { Acción }\end{array}$ & $\begin{array}{l}\text { El paciente realiza el cambio de conducta } \\
\text { necesarias para el cambio }\end{array}$ \\
\hline Mantenimiento & $\begin{array}{l}\text { Paciente activo en la consolidación del } \\
\text { cambio y la prevención de recaídas }\end{array}$ & $\begin{array}{l}\text { Reanudar los procesos de cambio } \\
\text { evitando que el cambio sea } \\
\text { estado previo de la acción }\end{array}$ \\
\hline
\end{tabular}

Consumo en los 30 días previos: se recomienda utilizar un orden lógico, conociendo la denominación de las diferentes drogas dentro de la jerga del paciente:

- Droga principal: en caso de que sea policonsumidor, se debe tener en cuenta que siempre habrá una sustancia que predomine en preferencia y en patrón de consumo.

- Drogas acompañantes: especificar si el consumo de las drogas se las hace todas juntas o por sustitución de la principal.

- Motivaciones para el consumo.

- Frecuencia del consumo y dosis: la declaración por el paciente suele ser superior a la real por miedo al síndrome de abstinencia y a no recibir las dosis adecuadas de psicofármacos en la desintoxicación.

- Vía de administración: es básica para conocer los factores de riesgo y las posibles complicaciones orgánicas.

- Patrón de consumo: especifica las condiciones en las que consume, si lo hace solo, en grupo, en eventos sociales, etc.

- Factores precipitantes: estados de ánimo, conflictos, estados de tensión y estrés, molestias físicas, disponibilidad económica, etc.

- Forma de obtener el dinero para la droga. 
- Tiempo de consumo nocivo: tiempo estimado desde que el consumo comenzó a convertirse en un problema, según la perspectiva del paciente.

- Consecuencias asociadas al consumo: físico, emocional, cognitivo, conductual y social.

- Comorbilidad física y/o psíquica: se debe apoyar con exámenes complementarios, útil sobre todo en infecciones prevalentes en este grupo de pacientes (VIH, hepatitis B, hepatitis $\mathrm{C}$, tuberculosis, enfermedades de transmisión sexual, etc.).
Periodo. Julio - Diciembre 2021

Vol. 5, Nro. 2, Publicado: 2021-12-31

- Conductas de riesgo para las enfermedades trasmisibles.

- Problemas socio-familiares: se debe tener énfasis en la estructura familiar y su funcionamiento, así como investigar sus repercusiones a nivel económico y laboral debido al consumo de drogas.

- Funcionamiento global del paciente: se resumirá la situación actual del paciente sobre las repercusiones de su consumo de drogas con respecto a todos los aspectos de la vida del paciente.

\section{Gráfico 2: Descripción del personal de salud, para una atención integral}

Desde esta perspectiva se contempla el consumo de drogas como un problema que afecta a la salud física, psíquica y relacional de las personas y que puede ser reducido mediante procedimientos educativos, para la salud es la disciplina que se encarga de transmitir conocimiento acerca de los comportamientos específicos que suponen un riesgo para la salud y sobre aquellos otros que, por el contrario, favorecen y protegen la salud.

\begin{tabular}{|c|c|c|}
\hline \multicolumn{3}{|c|}{ DESCRIPCIÓN DE ACTIVIDADES DEL PERSONAL DE SALUD } \\
\hline RESPONSABLE & ACTIVIDADES A DESARROLLAR & RECURSOS Y/O EQUIPOS \\
\hline $\begin{array}{l}\text { MEDICO } \\
\text { GENERAL }\end{array}$ & $\begin{array}{l}\text { Ingreso del usuario al sistema, definición de } \\
\text { diagnóstico inicial. Participación activa en el } \\
\text { proceso de recuperación y valoración continua. }\end{array}$ & $\begin{array}{l}\text { Consultorio médico con } \\
\text { dotación idónea, para toma de } \\
\text { signos vitales y valoración física } \\
\text { al usuario, computador, } \\
\text { software, recetarios, entre otros. }\end{array}$ \\
\hline $\begin{array}{l}\text { ENFERMERA } \\
\text { JEFE }\end{array}$ & $\begin{array}{l}\text { Actividades de apoyo que permitan determinar: } \\
\text { Estadio de cambio. } \\
\text { 1. Definición de problemas según la } \\
\text { gravedad de la adicción. } \\
\text { 2. Definir metas sobre el consumo, el } \\
\text { funcionamiento y la productividad. } \\
\text { 3. Acompañamiento permanente en el } \\
\text { proceso de rehabilitación. }\end{array}$ & $\begin{array}{l}\text { Sala de terapias con dotación } \\
\text { idónea, para toma de signos } \\
\text { vitales y valoración física al } \\
\text { usuario, computador, software, } \\
\text { entre otros. }\end{array}$ \\
\hline PSICOLOGO & 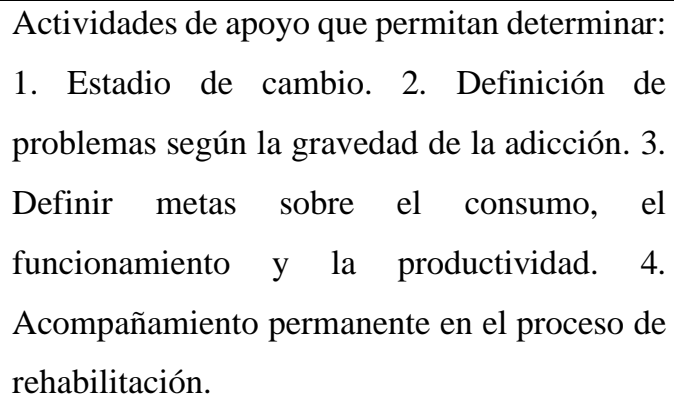 & $\begin{array}{l}\text { Consultorio independiente, Sala } \\
\text { de terapias con dotación idónea, } \\
\text { para toma de signos vitales y } \\
\text { valoración física al usuario, } \\
\text { computador, software, entre } \\
\text { otros. }\end{array}$ \\
\hline
\end{tabular}




\begin{tabular}{|c|c|c|}
\hline $\begin{array}{l}\text { TRABAJOR } \\
\text { SOCIAL }\end{array}$ & $\begin{array}{l}\text { Actividades de apoyo que permitan determinar: } \\
\text { 1. Estadio de cambio. } 2 \text {. Definición de } \\
\text { problemas según la gravedad de la adicción. } 3 . \\
\text { Definir metas sobre el consumo, el } \\
\text { funcionamiento y la productividad. } 4 . \\
\text { Acompañamiento permanente en el proceso de } \\
\text { rehabilitación. 5. Realizar monitoreo constante } \\
\text { a los usuarios, vía telefónica y visitas } \\
\text { domiciliarias }\end{array}$ & $\begin{array}{l}\text { Área de desarrollo Psicosocial. } \\
\text { Materiales de apoyo, cartillas, } \\
\text { folletos, volantes, registro de } \\
\text { asistencia, formatos y aplicación } \\
\text { de TEST, línea telefónica fija o } \\
\text { móvil. }\end{array}$ \\
\hline PSIQUIATRA & $\begin{array}{l}\text { Valoración de seguimiento, mediante consulta } \\
\text { externa. Formulación de medicamentos } \\
\text { pertinente a las necesidades de cada usuario. } \\
\text { Ejecución de actividades de apoyo Psicosocial. }\end{array}$ & $\begin{array}{l}\text { Consultorio médico con } \\
\text { dotación idónea, para toma de } \\
\text { signos vitales y valoración física } \\
\text { al usuario, computador, } \\
\text { software, recetarios, entre otros. }\end{array}$ \\
\hline
\end{tabular}

Tabla 3: Etapas de Atención Integral, fase 1-2-3

Atención integral residencial

Fase 1

Adaptación, motivación e inicio de proceso terapéutico

\section{Objetivo general}

Integrar al paciente al grupo de apoyo, mediante un proceso de adaptación, motivación e inicio de proceso Terapéutico.

\section{Objetivos específicos}

Recibir al paciente en un primer momento por parte de todos los intervinientes

- Brindar información sobre el servicio y sus normas internas, los tiempos de intervención, los objetivos del trabajo terapéutico y las posibilidades de tratamiento para cada usuario.

- Ampliar la evaluación del usuario en sus esferas psíquicas y orgánicas.

- Evaluar la situación familiar, social, educativa y laboral del usuario.

- Definir el acuerdo terapéutico e iniciar el proceso de tratamiento en comunidad.

- Firma del consentimiento informado por parte del área médica

\section{Actividades}

- Estrategias de Integración Social: A cargo del psicólogo y la trabajadora social, serán los responsables de realizar la red de apoyo de los pacientes.

- Inicio de Proceso Terapéutico: Introducción y socialización grupal e inicio de Terapias Psicológicas individuales, familiares y grupales, donde se asignan los líderes de la comunidad (de casa, aseo, deportes y académico) y de requerirse de terapia ocupacional.

- Realización de una planificación de las intervenciones del equipo de trabajo.

- Motivar al paciente, para el afianzamiento del compromiso de permanencia durante su proceso de recuperación. 
- Seguimiento del progreso terapéutico: Se realiza un análisis de casos respecto del desarrollo de las terapias en la reunión semanal del Equipo Técnico.

- Sesiones de Psicoeducación y Seminarios: Abordaje de temáticas inherentes al uso y consumo de drogas, a través de técnicas de interacción grupal.

- Terapias individuales y familiares que versen en torno a las características particulares del participante y su familia.

- Actividades terapéuticas, ocupacionales y recreativas.

Tiempo de duración: cuatro semanas

\section{Fase 2}

Integración, afianzamiento y esclarecimiento del proceso terapéutico

\section{Objetivo general}

Empoderar al paciente en su proceso terapéutico de cambio, con conciencia de sus características biopsico-sociales.

\section{Objetivos específicos}

- Recibir al paciente en un primer momento.

- Brindar información sobre el servicio y sus normas internas, los tiempos de intervención, los objetivos del trabajo terapéutico y las posibilidades de tratamiento para cada usuario.

- Ampliar la evaluación del usuario en sus esferas psíquicas y orgánicas.

- Evaluar la situación familiar, social, educativa y laboral del usuario.

- Definir el acuerdo terapéutico e iniciar el proceso de tratamiento en comunidad.

- Incluir a la familia dentro del proceso terapéutico, con el fin de comprender la problemática en torno al consumo y puedan apoyar al usuario en el proceso.

- Firma del consentimiento informado por parte del área médica

\section{Actividades}

Recepción de los participantes: Explicación empática por parte del Psicólogo y la trabajadora social acerca del programa, ambientación de los espacios, presentación del reglamento interno, firma del consentimiento informado.

- Estrategias de Integración Social: Trabajo social y el Psicólogo, serán responsables de realizar la red de apoyo del usuario.

- Inicio de Proceso Terapéutico: Introducción y socialización grupal e inicio de Terapias Psicológicas individuales, familiares y grupales, donde se asignan los líderes de la comunidad (de casa, aseo, deportes y académico) y de requerirse de terapia ocupacional y de gimnasio.

- Realización de una planificación de las intervenciones del equipo de trabajo.

- Motivar al participante para el afianzamiento del compromiso de permanencia durante su proceso de recuperación.

- Seguimiento del progreso terapéutico: Se realiza un análisis de casos respecto del desarrollo de las terapias en la reunión semanal del Equipo Técnico. 
- Desarrollo de terapias grupales con líneas terapéuticas acordes a las necesidades del grupo de participantes.

- Terapias individuales y familiares que versen en torno a las características particulares del participante y su familia.

- Asambleas multifamiliares con temáticas diseñadas en el equipo técnico y acordes a una línea terapéutica.

- Actividades terapéuticas, ocupacionales y recreativas.

Tiempo de duración: cuatro semanas

\section{Fase 3}

Fortalecimiento de los logros alcanzados y preparación para la reinserción socio-familiar.

\section{Objetivo general}

Brindar herramientas psicoterapéuticas de afrontamiento eficaces para la prevención de recaídas, la elaboración del proyecto de vida y la reinserción social, familiar y laboral.

\section{Objetivos específicos}

- Favorecer la identificación de factores personales de riesgo y de protección para prevenir recaídas en el consumo de drogas.

- Proveer de herramientas y estrategias cognitivas, afectivas y conductuales para afrontar los momentos de crisis.

- Formular un proyecto de vida que incluya metas de superación personal a corto, mediano y largo plazo.

- Establecer vínculos de apoyo familiar y social.

- Fortalecer las salidas terapéuticas hacia su núcleo familiar, incrementando su periodicidad cuando amerite.

- Determinar posibilidades de vinculación laboral y/o académica.

- Realizar un análisis de cada caso desde las distintas áreas profesionales dentro del tratamiento, para evaluar la extensión del proceso u otras alternativas.

\section{Actividades}

Grupos terapéuticos enfocados en la prevención de recaídas: Identificación de situaciones, pensamientos y/o estados emocionales para evitar nuevos consumos.

- Reconocimiento individual de situaciones de riesgo, basadas en la historia personal de cada sujeto, tanto en el ámbito grupal como individual.

- Construcción de alternativas de afrontamiento a situaciones de riesgo.

- Diseño del proyecto de vida del participante en conjunto con el área de trabajo social.

- Promoción de relaciones de apoyo familiares y sociales, con soporte de trabajo social. - Evaluación de las salidas terapéuticas realizadas y solventar las dificultades encontradas con el participante y la familia.

- Identificación de aspectos que puedan obstaculizar una separación saludable de la comunidad, para trabajar en ellos, de forma que se logre una adecuada adaptación a la sociedad. 
Hay que tener en cuenta que la vulnerabilidad no es binaria, sino multidimensional y relacional; no es unitaria, siempre hay graduaciones; no es estable, muta constantemente a lo largo del tiempo; las personas no son vulnerables, sino que están vulnerables. Los abordajes de reducción de vulnerabilidad han procurado ampliar el objetivo de las intervenciones desde el

\section{DISCUSIÓN}

Los resultados del estudio apoyan la importancia crítica de los factores de riesgo familiares económicos y biológicos, familiares para el desarrollo de abuso de sustancias en la juventud, características que ponen de manifiesto a la adolescencia como un período crítico para los efectos nocivos de la exposición al consumo de sustancias ilícitas. El desarrollo de estrategias de prevención e intervención inmediata dirigidas a los adolescentes con alto riesgo es imperante en este grupo (Martinez Loredo, 2016)

Así en este estudio se observa como la familia es el escenario donde la adolescente adquiere los recursos psicológicos con los que enfrentará esta etapa, estos recursos se traducen tanto en la capacidad para evaluar situaciones de la vida, esto es, anticipando y planeando la conducta a seguir para una mejor adaptación, como en la capacidad para reconocer y expresar sus reacciones afectivas. Si la estructura familiar no se desestabiliza o, si los padres no asumen su rol formativo de la manera más idónea, el individuo llegará a la adolescencia sin las herramientas necesarias para enfrentarse a la vida a través de la toma de decisiones correctas y convenientes. La estructura familiar juega un papel importante en el desarrollo del niño. (Loke Yoen \& Mak Wah, 2013) En el caso de las familias monoparentales, adolescentes y jóvenes que plano individual hacia el plano social. La actitud constructivista es la que mejor contribuye a que las personas puedan buscar y apropiarse de las informaciones que tengan sentido para ellas, movilizarse y hallar las alternativas prácticas que permitan superar las situaciones que las hacen vulnerables.

provienen de familias destructuradas tienen mayor probabilidad de caer en las drogas, ya que existe la insatisfacción de roles paternos lo que conlleva a una comunicación disminuida, la etapa adolescente, en la cual el estancamiento o la no superación de algunas instancias y de algunos estadios pueden llegar a generar el sentimiento de vulnerabilidad.

Por último, se puede ver de una manera tangible que la persona adicta no es capaz de cuidar de sí mismo, pues aquel trauma por decir así, se inició por la indefensión por parte sus padres, y ellos buscan sin saber la sujeción o suplantación de estos en búsqueda del objeto idea.

\section{Conclusiones}

- Proponer a la sociedad en su conjunto un análisis crítico, aunque pausado, objetivo y no moralizante de los usos y costumbres que legitiman el consumo de sustancias institucionalizadas. El comportamiento humano no es ajeno a las influencias de factores macrosociales que definen las características históricas, políticas y etnográficas de una sociedad. Los comportamientos cotidianos se asientan no sólo en decisiones individuales e influencias micro sociales, sino también en estos otros factores de amplio alcance. Por ello, no puede desdeñarse 
el conocimiento amplio sobre los condicionantes que llevan a una actitud acrítica ante las drogas de uso común que, por otra parte, son las que facilitan el aprendizaje en el consumo de sustancias.

- El modelo integral comunitario para el abordaje de los consumos problemáticos de drogas que se propone, es una respuesta diferente que surge frente a la ineficacia a pesar de los esfuerzos gubernamentales, de organizaciones de la sociedad y de los particulares, de las diferentes respuestas
Periodo. Julio - Diciembre 2021 Publicado: Vol. 5, Nro. 2, 2021-12-31 tanto preventivas como asistenciales que se han desarrollado hasta ahora. Sus principales dimensiones, integralidad, enfoque de las vulnerabilidades sociales y cuidados ampliados, en el fortalecimiento de lo singular y lo colectivo, la formación de redes, la promoción de la conciencia crítica y reflexiva, y el reconocimiento $y$ ejercicio de los derechos; perspectiva que considera que la toma de decisiones debe realizarse de modo compartido y redefine el rol del equipo profesional en función del diálogo con la comunidad.

\section{Referencias}

Francoises Contrera, Esguerra, G., Espinosa, J. C., Gutiérrez, C., \& Fajardo, L. (2006). Calidad de vida y adhesión al tratamiento en pacientes con insuficiencia renal crónica en tratamiento de hemodiálisis. Universitas Psychologica. Recuperado el 04 de 06 de 2021, de http://pepsic.bvsalud.org/scielo.php?script=sci_arttext\&pid=S1657-92672006000300005

Gómez Cobos, E. (2018). Adolescentes y familia. Revista Intercontinental de Psicología y Educacion, 12. Recuperado el 10 de 09 de 2021, de https://www.redalyc.org/pdf/802/80212387006.pdf

Guaranda Moreira , J. E. (2018). Consumo problemático de sustancias adictivas en adolescentes. Universidad Estatal del Sur de Manabí, ipijapa. Recuperado el 10 de 09 de 2021, de http://repositorio.unesum.edu.ec/bitstream/53000/1354/1/UNESUM-ECUADOR-

ENFERMERIA-2018-26.pdf

Sanz Turrado, M., Garrido Pérez , L., \& Caro Dominguez, C. (2017). Factores que influyen en la satisfacción del paciente de diálisis con enfermería. Scielo, 20(1). Recuperado el 08 de 06 de 2021, de https://scielo.isciii.es/scielo.php?script=sci_arttext\&pid=S2254-28842017000100009

Asociacion Panamericana de Psiquiatria. (2010). Manual de adicciones para psicólogos especialistas en psicología clínica en formación. España: SOCIDROGALCOHOL. Recuperado el 01 de 10 de 2021, de http://www.fundacioncsz.org/ArchivosPublicaciones/270.pdf

Bermeo Minchala, M. L., \& Jimenez Yora, J. V. (2014). Evaluacion de la calidad de vida de los pacientes con Insuficicencia Renal Cronica sometidos a hemodialisis. Azoguez. Recuperado el 04 de 06 de 2021, de http://dspace.ucuenca.edu.ec/bitstream/123456789/21563/1/Tesis\%20Pregrado.pdf

Cid Monckton, P., \& Pedrão , L. J. (2011). Protective and family risk factors related to adolescent drug use. Revista Latino-Americana de Enfermagem, 15. Recuperado el 10 de 09 de 2021, de https://doi.org/10.1590/S0104-11692011000700011 
Consejo de Organizaciones Internacionales de las Ciencias Medicas. ( para la investigacion relacionada con la salud con seres vivos. Organizacion Mundial de la Salud, Ginebra. Recuperado el 3 de 06 de 2021, de https://iris.paho.org/bitstream/handle/10665.2/34457/9789290360902spa.pdf?sequence $=5 \&$ isAllowed $=\mathrm{y}$

David, R. G., \& Sanjuán Hernández, D. (2018). Satisfacción percibida por los pacientes en el servicio de hemodiálisis del hospital general de La Palma. Redyalic, 15. Recuperado el 2021 de 07 de 28, de https://www.redalyc.org/pdf/3598/359833150002.pdf

Instituto Provincial de Bienestar Social de Cordoba. (2018). Un Modelo de Getiona para la Promocion de la Salud y la Prevencion y las Adicciones en la Provincia de Cordova. Cordoba. Recuperado el 30 de $09 \quad$ de $2021, \quad$ de http://www.ipbscordoba.es/uploads/Documentos/Modelo_gestion_adicciones_2011.pdf

Jimenez, T. I., Murgui, S., Estevez, E., \& Musitu, G. (2007). Comunicacion familiar y comportamientos delictivos en adolescentes de Madrid, el doble rol de mediador. Redialyc, 12. Recuperado el 10 de 09 de 2021, de https://www.redalyc.org/pdf/805/80539303.pdf

Loke Yoen, A., \& Mak Wah, Y. (2013). El proceso familiar y las influencias de los compañeros sobre el consumo de sustancias por parte de los adolescentes. Revista internacional de investigación ambiental y salud pública, 15. Recuperado el 13 de 09 de 2021

Martinez Loredo, V. (2016). Parenting styles and alcohol use among adolescents:. European Journal of Investigation in Health, Psychology and Education, 9. Recuperado el 13 de 09 de 2021

Municipio de Medellin- Ministerio de Salud. (2014). Abordaje integral para la intervención de conductas adictivas. Medellin, Colombia. Recuperado el 30 de 09 de 2021, de http://www.odc.gov.co/Portals/1/publicaciones/pdf/consumo/recursos/CO31052016-manualbordaje.Integral-intervencion-conductas-adictivas.pdf

Observatorio de Drogas de Colombia. (09 de 2018). Observatorio de Drogas Colombia. Recuperado el 10 de 09 de 2021, de http://www.odc.gov.co/PUBLICACIONES/ArtMID/4214/ArticleID/5929/Informe-MundialSobre-las-Drogas-2014

OPS. (10 de 03 de 2018). Organizacion Panamericana de la Salud. Recuperado el 30 de 09 de 2021, de https://www3.paho.org/hq/index.php?option=com_content\&view=article\&id=10542:2015opsoms-sociedad-latinoamericana-nefrologia-enfermedad-renal-mejorartratamiento\&Itemid=1926\&lang=es

Ponce Bacusoy, M. T., Suarez Martinez, F. J., \& Pincay Parrales, E. G. (2021). Trastornos del comportamiento asociados a sustancias psicoactivas en estudiantes de bachillerato de la Unidad Educativa Alejo Lazcano. Revista Ciencias, 16. doi:http://dx.doi.org/10.23857/dc.v7i1.1867

Sánchez Hernández, C., Rivadeneyra Espinoza, L., \& Aristil Chery, P. M. (2016). Calidad de vida en pacientes bajo hemodiálisis en un hospital público de Puebla, México. Scielo, 20(3), 15. Recuperado el 10 de 08 de 2021, de http://scielo.sld.cu/scielo.php?pid=S102502552016000300006\&script=sci_arttext\&tlng=en 
Tena Suck, A., Castro Martínez, G., Marín Navarrete, R., Gómez Romero, P., \& De la Fuente-Martín, A. (2018). Consumo de sustancias en adolescentes: consideraciones para la práctica médica. Scielo, 11. Recuperado el 04 de 10 de 2021, de http://www.scielo.org.mx/scielo.php?script=sci_arttext\&pid=S018648662018000200008\&lang=pt

Tumbaco Quimis, G. A. (2018). Consumo problemático de sustancias adictivas en los adolescentes. Jipijapa. Recuperado el 0910 de 2021, de http://repositorio.unesum.edu.ec/bitstream/53000/1347/1/UNESUM-ECUADOR-

ENFERMERIA-2018-19.pdf 